\title{
Critical arrhythmia in postoperative cardiac children: Recognition and management
}

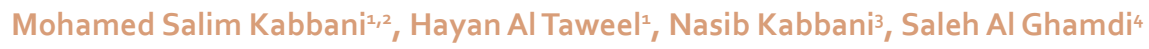 \\ ${ }^{1}$ Department of Cardiac Science, Division of Pediatric Cardiac Critical Care Medicine, King Abdulaziz Medical City, Ministry of National Guard \\ Health Affairs, ${ }^{2}$ King Saud Bin Abdulaziz University for Health Sciences, ${ }^{3}$ College of Medicine, Alfaisal University, ${ }^{4}$ Department of Cardiac \\ Science, Division of Pediatric Cardiology, King Abdul Aziz Medical City, Ministry of National Guard Health Affairs, \\ Riyadh, Kingdom of Saudi Arabia
}

\begin{tabular}{|c|}
\hline Access this article online \\
\hline Website: www.avicennajmed.com \\
\hline DOI: 10.4103/ajm.AJM_14_17 \\
\hline Quick Response Code: \\
\hline
\end{tabular}

\section{ABSTRACT}

Arrhythmias after pediatric cardiac surgery are common and can be life-threatening. They occur intraoperatively or may appear shortly after surgery during postoperative care. They require early management and specific intervention. In this review, we describe important critical arrhythmias that are encountered during postoperative management of children undergoing cardiac surgery. We review the diagnosis, management, and explain the role of epicardial electrocardiogram in diagnosing certain types of postoperative rhythm abnormalities seen during early period after pediatric cardiac surgery.

Key words: Bradycardia, critical arrhythmia, pediatric cardiac surgery, tachycardia

\section{INTRODUCTION}

Postoperative arrhythmias are common and occur in $7.5 \%$ up to $48 \%$ of postoperative pediatric cardiac patients. ${ }^{[1,2]}$ They may occur intraoperatively as a result of direct injury or manipulation to the cardiac conduction system or during the early postoperative period as a result of local tissue edema and inflammation in the myocardium adjacent to conduction system. Additional contributing factors are arrhythmogenic effects of drugs used commonly during postoperative care and electrolyte disturbances encountered after cardiac surgery. The majority of arrhythmias encountered early after surgeries are self-limited or transient and recover with proper management. ${ }^{[3]}$

The etiology is multifactorial. It is important to recognize that cardiac surgery and cardiopulmonary bypass (CPB) may affect myocardium leading to "stunned myocardium" with depressed function. ${ }^{[4]}$ The heart muscle and conduction system are sensitive during the postoperative period and require optimal hemostasis for recovery

Address for correspondence: Dr. Mohamed Salim Kabbani, Department of Cardiac Science, Division of Pediatric Cardiac ICU, MC 1423, King Abdulaziz Medical City, P.O. Box: 22490, Riyadh 11426, Kingdom of Saudi Arabia.

E-mail: kabbanim@ngha.med.sa and rhythm stabilization. ${ }^{[5]}$ Electrolytes disturbances, acidosis, hypotension, and hypoxia are irritants and provoke arrhythmia that needs to be corrected promptly. In particular, $\mathrm{Mg}^{+}, \mathrm{K}^{+}$, and $\mathrm{Ca}^{++}$are essential cations for normal contractility and cardiac rhythm stability. ${ }^{[5]}$ CPB may lead to significant arrhythmogenic electrolytes disturbances, ${ }^{[5,6]}$ and it was reported to be a risk factor for postoperative arrhythmia. ${ }^{[1,7]}$ Correcting electrolytes abnormalities and optimizing their serum levels help to stabilize the heart and reduce further irritation and excitation of the conduction system. ${ }^{[8,9]}$ These measures are fundamental during postoperative management of any electrical rhythm disturbance postcardiac surgery. Many clinically significant arrhythmias, however, are not related to electrolyte disturbances, and unless, they are controlled or managed rapidly, they can lead to significant clinical deterioration or even mortality. Studies have demonstrated certain risk factors for the development of

This is an open access article distributed under the terms of the Creative Commons Attribution-NonCommercial-ShareAlike 3.0 License, which allows others to remix, tweak, and build upon the work non-commercially, as long as the author is credited and the new creations are licensed under the identical terms.

For reprints contact: reprints@ @medknow.com

Cite this article as: Kabbani MS, Al Taweel H, Kabbani N, Al Ghamdi S. Critical arrhythmia in postoperative cardiac children: Recognition and management. Avicenna J Med 2017;7:88-95. 
early postoperative arrhythmias in children undergoing cardiac surgeries that include younger age and longer bypass and cross-clamp times. ${ }^{[2]}$ In addition, the repair of atrioventricular (AV) septal defects was found to be an independent risk of early postoperative arrhythmias in children. ${ }^{[2]}$

In general, arrhythmia occurring during the postoperative period can be classified into bradyarrhythmia (slow rhythm) or tachyarrhythmia (fast rhythm). Furthermore, tachyarrhythmia can be divided based on the shape of QRS complex to narrow QRS complex tachyarrhythmia $(\leq 0.09$ s) or wide QRS complex tachyarrhythmia $(>0.09 \mathrm{~s}) .{ }^{[10]}$

Temporary pacemaker is important in the management of arrhythmias after cardiac surgery, and it is life-saving in cases of bradyarrhythmia. As such cardiac surgeons place temporary "atrial and ventricular" or "ventricular" pacing wires intraoperatively for good number of children undergoing heart surgery. The presence of atrial and ventricular pacing wires can help not only in arrhythmias management but also in diagnosing certain type of arrhythmias by performing epicardial atrial electrocardiogram (ECG). ${ }^{[1]}$

In this review, we will discuss common rhythm disturbances seen after pediatric cardiac surgery from critical care perspective. To facilitate their evaluation, we classify postoperative arrhythmias into:

- Tachyarrhythmia: These can be further categorized into

a. Narrow QRS complex tachycardia

b. Wide QRS complex tachycardia

- Bradyarrhythmia: These can be further categorized into

a. Sinus bradycardia and sinus node dysfunction

b. Heart block and ventricular asystole.

We will discuss the incidence of each type of arrhythmias in postoperative pediatric cardiac surgery, when do we see them, what are the risk factors, and how can we recognize their different types. In addition, we will discuss how can we do and interpret epicardial ECG to facilitate diagnosis and management of postoperative arrhythmias.

\section{TACHYARRHYTHMIA POSTPEDIATRIC CARDIAC SURGERY}

Tachyarrhythmia is commonly encountered after pediatric cardiac surgery. In general, it can be classified according to their origin into narrow QRS complex $(\leq 0.09 \mathrm{~s})$ or wide QRS complex tachycardia (>0.09 s).
Narrow QRS complex tachyarrhythmia after pediatric cardiac surgery

Sinus tachycardia

Sinus tachycardia is not an arrhythmia per se, but when the rhythm is fast, it may not be easy to differentiate sinus tachycardia from atrial tachycardia or junctional ectopic tachycardia (JET). It is characterized by fast regular rhythm with $\mathrm{P}$ wave preceding each QRS complex. The RR intervals are equal to PP intervals. The heart rate in sinus tachycardia is variable according to age and usually ranges between 160 and $200 \mathrm{bpm}$ and rarely exceeds $200 \mathrm{bpm}$ in infants. The QRS complex is narrow ( $\leq 0.09 \mathrm{~s}$ ) while RR intervals are equals and regular. There are many causes for sinus tachycardia after cardiac surgery including fever, anemia, pain, the use of catecholamines, hypovolemia, hypoxia, low cardiac output state, systemic inflammatory response syndrome, and many others. The management of sinus tachycardia is based on treating the cause. Maintaining adequate preload and normal hemoglobin level can help treating tachycardia associated with hypovolemia or anemia during postoperative management.

\section{Junctional ectopic tachycardia}

It is considered to be the most common type of tachyarrhythmia seen during early postoperative care and encountered in $2 \%-11.2 \%$ of children undergoing cardiac surgery. ${ }^{[12]}$ JET is life-threatening tachyarrhythmia that happens particularly after procedures in proximity to $\mathrm{AV}$ node (AVN) such as tetralogy of Fallot or perimembranous ventricular septal defect or atrioventricular septal defect repair. ${ }^{[12,13]}$ It is an automatic focus firing in its own that may start spontaneously during surgery or immediately after surgery most commonly during the first postoperative day. The heart rate is usually between 170 and 260 with narrow QRS complex and AV dissociation. ${ }^{[13]}$ The focus of the fast rhythm in JET originates within or near AVN and impulses are conducted at the same time retrograde to atria and antegrade to ventricles leading to dissociation between atrial and ventricular conduction. Occasionally, ventricular conduction and rhythm can be even faster than atrial rhythm. Hence, the atrial activation and $\mathrm{P}$ wave may come before, after, or buried within QRS complex since the ventricular activation is independent and not related to atrial activation. Most of the time atrial $\mathrm{P}$ waves is absent and cannot be seen in the regular ECG [Figure 1a]. Hemodynamically and because of fast junctional rhythm, the ventricle cannot fill up normally during diastole. This is in addition to the lack of proper sequential synchronization between atrial contraction and ventricular diastolic relaxation that permits proper ventricular diastolic filling and optimal cardiac output; it is well known that atrial 
contractions contribute to approximately $25 \%$ of ventricular filling, and hence, cardiac output in the postoperative period. ${ }^{[14]}$ Losing atrioventricular synchronization and "atrial kicks" may have detrimental effects on recently operated sick heart leading to inadequate cardiac output and profound shock.

\section{Diagnosis of junctional ectopic tachycardia}

Diagnosis of JET is not always easy as it resembles other supraventricular tachycardia (SVT). SVT, however, responds commonly to adenosine and cardioversion while JET is usually refractory to both of them. Depending on the site of the foci that generates the JET, P wave may appear inverted due to retrograde activation of the atrium and may be seen after QRS or before the QRS if the foci of JET activating atrium before the ventricle. If the focus of retrograde conduction is blocked, then no retrograde $\mathrm{P}$ wave is seen; hence, the atrium is activated by normal sinus beat, and typically, a normal $\mathrm{P}$ wave morphology appears marching through junctional rhythm without a clear relation to QRS due to failure to penetrating to ventricle, it may precede, follow, or be hidden within the QRS complex.

We can recognize two patterns of conductions: ventricular-atrial (VA) association and VA dissociation. In VA association, every ectopic beat will be conducted to ventricle and atrium with 1:1 conduction ratio. On the other hand, JET is more commonly occurring with VA dissociation where impulses will be conducted to ventricle faster and more often than atrium. Therefore, ventricle to atrium contractions ratio will be higher in VA dissociation more than the 1:1 ratio seen in VA association. ${ }^{[12]}$ This is a key diagnostic feature.
The presence of atrial pacing wire is very helpful in making the diagnosis of JET by differentiating it from other types of SVT. The atrial wire is simply attached to one ECG leads and 12 leads ECG record is then obtained in the usual fashion, it can demonstrate better than regular ECG the location of $\mathrm{P}$ wave in relation to QRS complex and shows dissociation pattern. When atrial pacing wires are connected to lead (I) or (V2 and V5) as demonstrated in [Figure 2], the P wave is accentuated and "magnified" and appears as sharp spike which can be easily visualized and can be identified with its exact position whether before, within or after QRS complex compared to standard ECG tracing. The appearance of $\mathrm{P}$ wave within or immediately after QRS complex is supportive to diagnosis of JET [Figure 1a-d].

\section{Management of junctional ectopic tachycardia}

There are common recommendations in the management of JET that include avoidance of inotropes or other medications that enhance tachycardia, optimizing electrolytes, correction of anemia, acidosis, and hypoxia, adequate sedation and analgesia, maintaining good preload, and cooling the core temperature to $34^{\circ} \mathrm{C}-35^{\circ} \mathrm{C}$. Amiodarone is a Class III antiarrhythmic agent and has the highest efficacy rate for the treatment of JET. ${ }^{[15]}$ It, is therefore, the first-line treatment option to restore sinus rhythm and slow the heart rate. Amiodarone should be given whenever JET leads to hemodynamic instability, and it is effective in $80 \%-90 \%$ of cases. ${ }^{[12]}$ The recommended dose is $5 \mathrm{mg} / \mathrm{kg}$ over $2 \mathrm{~h}$ followed by infusion of $10-20 \mathrm{mg} / \mathrm{kg} / 24$ up to $46 \mathrm{~h} \cdot{ }^{[15,16]}$ Recently, dexmedetomidine, a selective $\alpha$ - 2 adrenoceptor agonist used for sedation, has been reported to reduce the incidence of JET and have increased success in the management of JET in

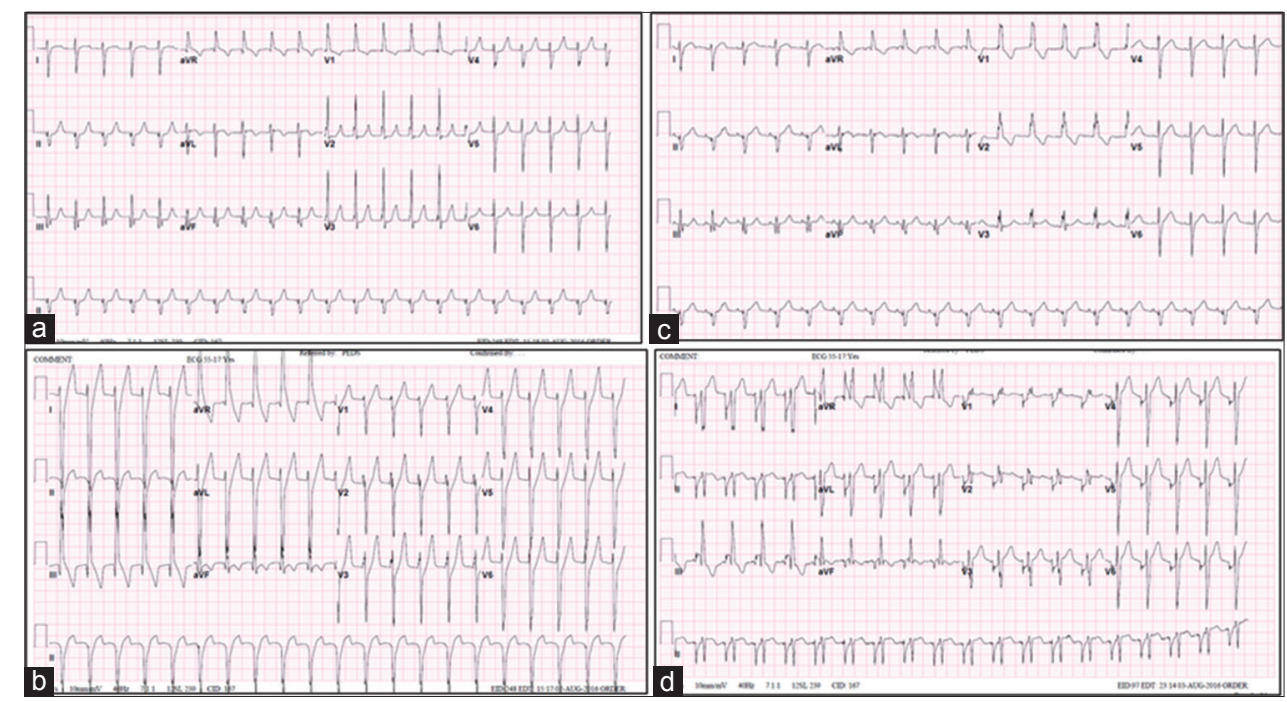

Figure 1: (a) Regular electrocardiogram in postoperative patient with tachycardia and absent $P$ waves. (b) Epicardial atrial electrocardiogram with atrial pacing wires connected to lead I. Electrocardiogram is performed after regular electrocardiogram (a) demonstrating junctional rhythm with P wave buried in QRS complex. (c) Regular electrocardiogram in same patient after $24 \mathrm{~h}$ of management with the return of normal sinus rhythm. (d) Epicardial atrial electrocardiogram for the same patient after 24 demonstrating normal sinus rhythm. P wave appears now magnified and normally preceding each QRS complex 
children. It modulates the release of catecholamine resulting in bradycardia and hypotension. Due to its depressive effects on AVN, dexmedetomidine appears to be helpful in slowing the JET rhythm and reestablishing normal sinus rhythm in postoperative patients with JET. In the prospective randomized study, dexmedetomidine infusion of $0.75 \mu \mathrm{g} / \mathrm{kg} / \mathrm{h}$ was found to decrease the incidence of JET after tetralogy of Fallot repair. ${ }^{[17]}$ Another study reported a significant decrease in the incidence of ventricular and supraventricular tachyarrhythmia, including JET with the use of peroperative dexmedetomidine in children undergoing cardiac surgery. ${ }^{[18]}$ We propose our Institutional General Guidelines for the management of JET:

- Correct electrolytes disturbance, target serum $\mathrm{Mg}^{+}$level between 1.3 and 1.5

- Treat fever and maintain core temperature initially at $35^{\circ} \mathrm{C}$

- Minimize inotropes as much as possible

- Correct hypoxia, acidosis, and maintain good preload

- Correct anemia and target hemoglobin of 12-14

- Optimize analgesia and sedation and start dexmedetomidine with loading dose of $0.1 \mathrm{mg} / \mathrm{kg}$ followed by infusion $(0.5-0.7 \mu \mathrm{g} / \mathrm{kg} / \mathrm{h})$

- If patient is hemodynamically unstable, start loading dose of amiodarone $(5 \mathrm{mg} / \mathrm{kg}$ ) over $2 \mathrm{~h}$ followed by continuous infusion of $10-20 \mathrm{mg} / \mathrm{kg} /$ day for another $46 \mathrm{~h}$

- If the patient did not respond to the previous measures, cool core temperature further to 34 and consider "reverse sequential" pacing, where dual chamber pacemaker with atrial and ventricular pacing temporary wires are connected to pacer box in reverse fashion (atrial lead in ventricular channel and vice versa) with increased AV delay and shorten postventricular atrial refractory period. The reverse ventricular and atrial wire leads target

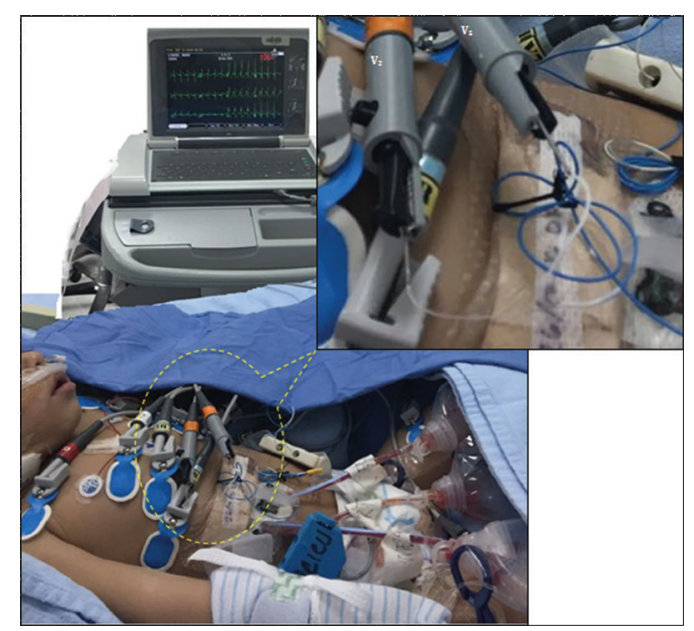

Figure 2: Connection of 2 atrial pacing wires to V2 and V5 leads then conducting 12 leads electrocardiogram to obtain atrial epicardial electrocardiogram better synchronization through the attempt of ventricle sensing and atrial pacing. The aim of this measure is to maintain stable hemodynamic by recruiting atrial kick with sequential pacing while antiarrhythmic medication is persuade as a bridge until tachycardia is controlled

- Consider ECMO for refractory cases with life-threatening refractory rhythm.

Of note that rapid atrial pacing may be considered early in many institution even before starting the amiodarone.

\section{Nodal-dependent supraventricular tachycardia}

There are two forms of nodal-dependent tachycardia, the so-called AV reentrant tachycardia (AVRT) and AV nodal reentry tachycardia (AVNRT). The former involves abnormal accessory pathway connecting ventricle to atrium retrograde and $\mathrm{AVN}$ as antegrade limb to maintain reentrant mechanism of tachycardia. AVNRT is confined to compact AVN produces a mechanism of reentrant due to two pathways within AVN, one is fast and the other is slow conducting pathway acting as antegrade and retrograde limb of tachycardia. SVT usually starts suddenly and responds most of the time to adenosine [Figure $3 \mathrm{a}$ and $\mathrm{b}$ ]. ECG is characterized by narrow complex, fast, and regular tachycardia with clear visible inverted retrograde P-wave following the preceding QRS in case of AVRT while no visible $\mathrm{P}$ wave that can be appreciated with AVNRT. Cardiac surgery can trigger existing mechanism for SVT which is relatively easy to control compared to automatic focus; however, SVT that do not respond to adenosine, synchronized cardioversion can be used at $0.5-1 \mathrm{~J} / \mathrm{kg}$. In refractory cases that do not respond to adenosine or cardioversion, second line of the management such as amiodarone or short-acting b-blocker infusion (esmolol) can be considered. ${ }^{[19,20]}$

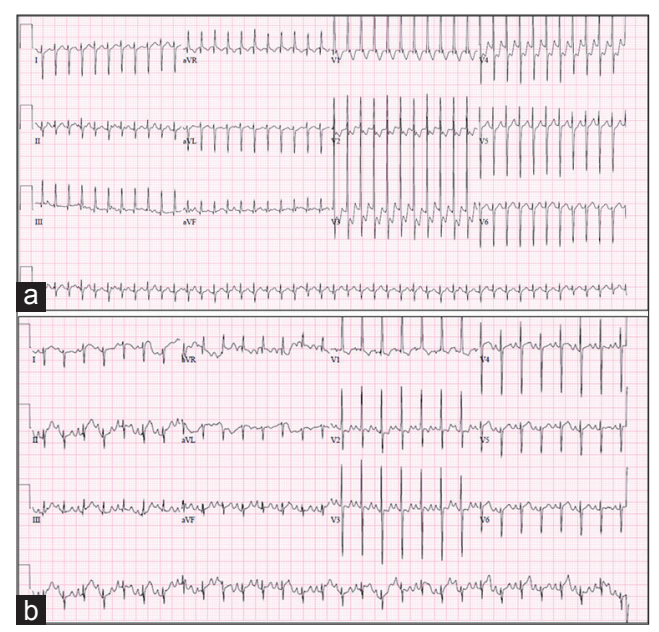

Figure 3: Supraventricular tachycardia after coarctation repair in neonate (a) before adenosine, (b) conversion to sinus rhythm after adenosine 


\section{Atrial flutter}

Atrial flutter is reentrant tachycardia confined to atrium, it is unusual tachycardia after pediatric cardiac surgery, characterized by saw teeth $\mathrm{P}$ waves at rate more than 300/min with narrow QRS complex. There is usually AV block (AVB) with atrial to ventricular conduction ratio of $2-4: 1$. The RR interval is variable. This type of tachycardia can be confused with SVT or JET when conducting 1:1 over the AVN. One-way to differentiate this type of rhythm is by giving adenosine while obtaining ECG tracing. Adenosine blocks conduction at the AVN and unmask the atrial $\mathrm{P}$ wave demonstrating the actual atrial rate and the classical "saw teeth" appearance of $\mathrm{P}$ waves characteristic of atrial flutter [Figure 4a]. Atrial flutter that affects hemodynamic responds usually to synchronized cardioversion at $0.5-1 \mathrm{~J} / \mathrm{kg}$, similar to all kinds of reentrant tachycardia; cardioversion will help converting abnormal rhythm to sinus [Figure 4a-c].

\section{Atrial fibrillation}

Atrial fibrillation is a rare arrhythmia after pediatric cardiac surgery. The rhythm is irregularly irregular; there are no discernible $\mathrm{P}$ waves with atrial rate more than $350 / \mathrm{min}$. QRS complex is narrow with variable RR intervals. The fast rhythm may cause hemodynamic instability. The management is targeted either to rate control or rhythm control. Drugs that slow heart rate by blocking AVN ultimately result in reasonable ventricular response and restore stable hemodynamics, including beta-blocker, calcium channel blockers, and digoxin. Rhythm control can be achieved chemically by antiarrhythmic medication such as amiodarone or electrically by synchronized cardioversion. This rhythm as well as flutter predisposes to clot formation if lasted for more than $72 \mathrm{~h}$, so echocardiography should be

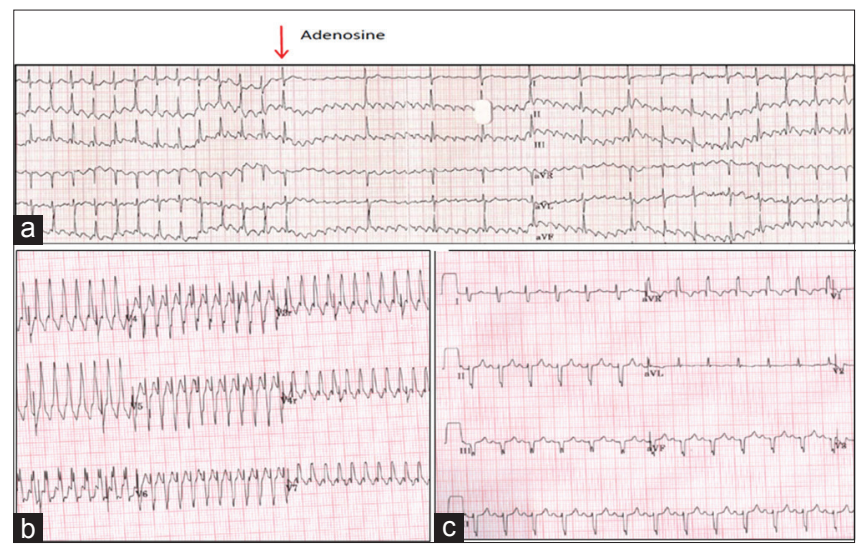

Figure 4: (a) Atrial flutter presenting as fast atrial rhythm that is indistinguishable from other narrow QRS complex tachyarrhythmia such as supraventricular tachycardia or junctional tachycardia. The flutter saw-teeth P waves became unmasked and apparent after administration of adenosine. (b) Electrocardiogram tracing for the same patient demonstrating fast flutter rhythm before synchronized cardioversion, then (c) electrocardiogram demonstrates normal sinus rhythm after synchronized cardioversion performed before cardioversion to rule out the presence of thrombi in the atria.

\section{Wide QRS complex tachyarrhythmia after pediatric cardiac surgery}

In general, this type of arrhythmia is life-threatening and requires immediate recognition and management. In this regard, we can recognize three important rhythms abnormalities that include ventricular tachycardia, supraventricular tachycardia with aberrancy, and ventricular fibrillation.

\section{Ventricular tachycardia}

Ventricular tachycardia is life-threatening fast organized rhythm with wide QRS complex. It is often associated with hemodynamic instability or even absent pulse. If there is hemodynamic instability but with palpable pulse, the management required synchronized cardioversion starting with $0.5 \mathrm{~J} / \mathrm{kg}$ followed by the infusion of amiodarone or lidocaine. ${ }^{[10]}$ On the other hand, ventricular tachycardia with absent pulse required cardiopulmonary resuscitation (CPR) with immediate defibrillation starting with $2 \mathrm{~J} / \mathrm{kg}$ as soon as the defibrillator is set and charged [Figure 5a and b]. Amiodarone or lidocaine infusion should also be given after defibrillation to prevent further attacks.

\section{Supraventricular tachycardia with aberrancy}

SVT in association with the right or left bundle branch block may appear as wide QRS complex tachycardia with morphology that is difficult to differentiate from ventricular tachycardia. Epicardial ECG with atrial leads recording may help differentiating the origin of the tachycardia. Trial of adenosine can also help in the management of SVT.

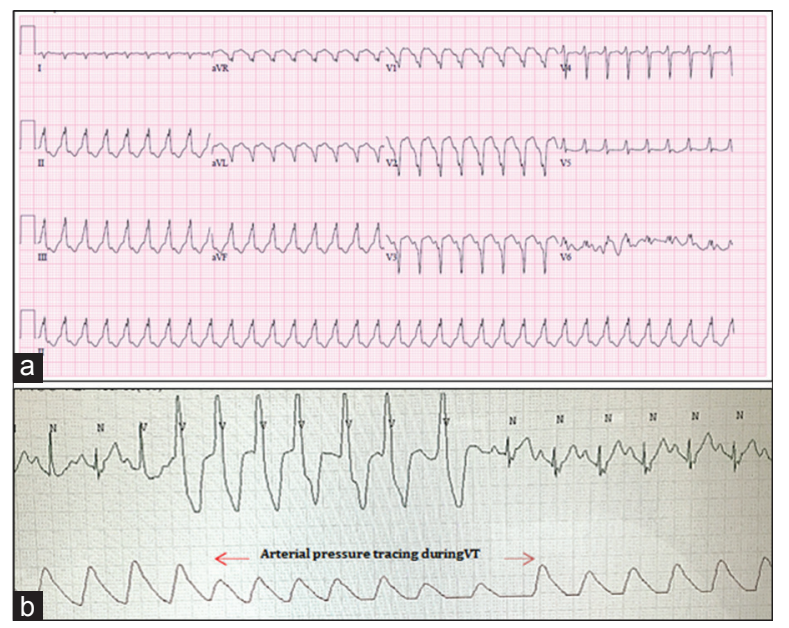

Figure 5: (a) Twelve leads electrocardiogram of postoperative patient who developed ventricular tachycardia with pulse. (b) Short run of ventricular tachycardia associated with decrease blood pressure as demonstrated by arterial line tracing 
If the child is hemodynamically unstable synchronized cardioversion may be necessary.

\section{Ventricular fibrillation}

This is life-threatening rhythm required immediate CPR with defibrillation starting with $2 \mathrm{~J} / \mathrm{kg}$ up to $10 \mathrm{~J} / \mathrm{kg}$ [Figure 6]. During postoperative care, this may happen as a primary sudden isolated cardiac event or may happen secondary to respiratory or hemodynamic failure. It is often triggered by catastrophic events leading to ventricular fibrillation and subsequently cardiac arrest. The cause for the arrest should be treated immediately. If there is no obvious reversible cause and the child does not respond promptly to initial resuscitation measures the surgeon, anesthesia, operative staff, and ECMO team should be immediately involved for sternal reopening and deployment of extracorporeal mechanical life support. If there is standby ECMO team, the team and cardiac surgeon should be immediately alerted and get prepared once life-threatening arrhythmia is encountered in any fresh postoperative cardiac case.

\section{BRADY ARRHYTHMIA AFTER PEDIATRIC CARDIAC SURGERY}

\section{Sinus bradycardia and sinus node dysfunction}

Sinus bradycardia is common after pediatric cardiac surgery. It is recognized as regular rhythm with $\mathrm{P}$ wave before each QRS complex and slow heart rate. There are multiple reasons for sinus bradycardia after cardiac surgeries that include hypothermia, deep sedation, and medications. The management of bradycardia is by treating the cause. It is important to closely monitor the signs of adequate cardiac output in cases of bradycardia after cardiac surgery as cardiac output is equal to stroke volume multiplies by heart rate. Infants have stiff ventricle and depends on increasing heart rate rather than stroke volume to increase their cardiac output. When a child has low heart rate postoperatively, the cardiac output may not meet body $\mathrm{O}_{2}$ demand with resultant anerobic oxygenation and tissue hypoxia. The etiology of sinus bradycardia sometimes may not be clear and it is labeled as sinus node dysfunction. The management may require temporary pacing of the heart at higher rate to increase cardiac output and tissue perfusion.

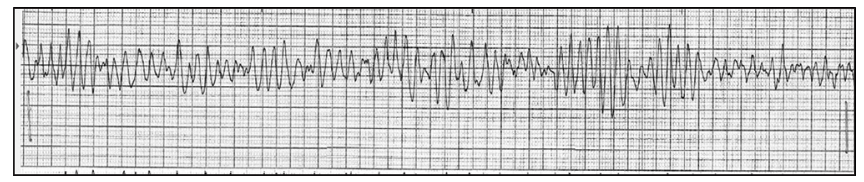

Figure 6: Ventricular fibrillation in 2 years old infant developed $2 \mathrm{~h}$ after cardiac repair
Atrial pacing Atrial Pacing, Atrial Sensing, Response to Sensing by Inhibition (AAI) is the optimal mode for pacing symptomatic bradycardia associated with intact AV nodal conduction. Other mode such as Ventricle Pacing, Ventricle Sensing, Response to Sensing by Inhibition VVI can also be used if there is only ventricular wire. Normal sinus rhythm usually returns after treating the cause or spontaneously recovered within few days following cardiac repair.

\section{Sinus node dysfunction}

Sick sinus syndrome or sinus node dysfunction is a group of heart rhythm disorders that can be encountered sometimes after pediatric cardiac surgery. It is usually seen after heart surgery involving the right atrium near sinus node. Direct injury or manipulation of tissue near sinus atrial node is linked to this syndrome as in cases of atrial septal defect closure or partial anomalous pulmonary venous connection repair. ${ }^{[21,22]}$ It is also reported after the Fontan procedure. It may appear as slow sinus rhythm that may alternate with episodes of tachycardia-bradycardia or may appear as frequent sinus pauses or may appear as sinus arrest. The slow abnormal rhythm will lead to decrease cardiac output that does meet body demand. In sick sinus node, the conduction through AVN remains intact and patient responds to temporary atrial pacing (AAI). Sinus node dysfunction usually improves with time; however, in cases where rhythm does not return back to normal, a permanent pacemaker is indicated. ${ }^{[22]}$

\section{Heart block and ventricular asystole Heart block}

Partial and complete AVB are the most common indication for permanent pacemaker need postpediatric cardiac

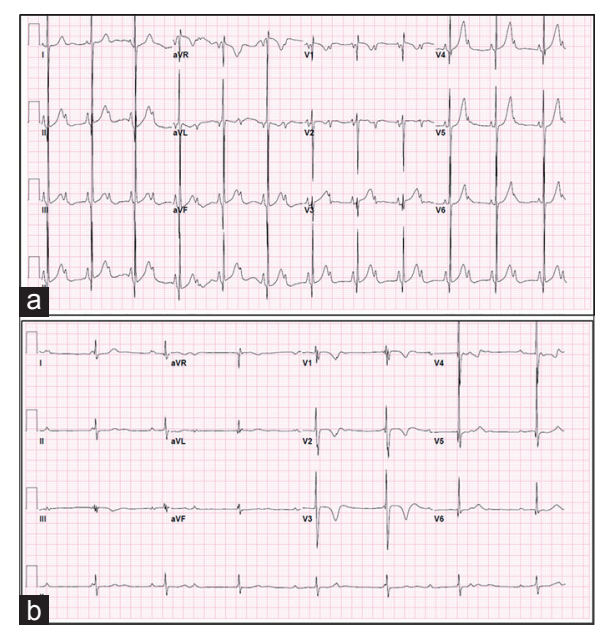

Figure 7: (a) Postoperative child with second-degree heart block. (b) Third-degree heart block after atrioventricular septal defect repair demonstrating $P$ waves "marching through" QRS complexes 
surgery. ${ }^{[20]}$ Heart block is encountered during or after cardiac surgery near AVN such as tetralogy of Fallot repair, atrioventricular septal defect repair, or perimembranous ventricular septal defect repair. ${ }^{[23]}$ There are certain types of patients at risk for AVB such as patients with congenitally corrected transposition of the great arteries due to the location and development of conduction system in this disease. ${ }^{[24]}$ Children who have heart block endure slow rhythm with dyssynchrony between atrial and ventricular contractions. That may negatively affect cardiac output leading to hypoperfusion with hemodynamic instability. Symptomatic postoperative children with Mobitz Type II second-degree heart block or third-degree heart block require temporary pacing [Figure $7 \mathrm{a}$ and $\mathrm{b}$ ]. If patient has atrial and ventricular wires, then dual chamber pacing is indicated using dual chamber pacing mode such as Dual Pacing, Dual Sensing, Dual Inhibition and Triggering (DDD) or Dual Pacing, Dual Sensing, Dual Inhibition (DDI). If patient has only ventricular pacing wire, then single chamber pacing with VVI mode (ventricular sensing, ventricular pacing, and ventricular inhibition) is indicated. Many children improve and recover within 7-10 days after surgery and reclaim their own baseline normal sinus rhythm with normal AV conduction. Others may continue to have heart block with slow rhythm that requires permanent pacemaker insertion.

\section{Venticular asystole}

When a patient develops heart block the sinus node impulses cannot be conducted through AV node. A lower escape rhythm in cardiac conduction system may take over instead of sinoatrial node. The escape rhythm has usually slower rate than normal sinus rhythm and mislays sequential synchronization between atrium and ventricle leading to inadequate cardiac output. Infrequently, the heart with complete AVB exhibits no escape rhythm with complete heart block and ventricular asystole or standstill. The child with postoperative ventricular asystole depends entirely on pacemaker for cardiac output. Steroid, theophylline, atropine, and isoproterenol have been tried to stimulate ventricular escape rhythm and AV mode conduction. Many children recover and return to normal sinus rhythm with normal AV conduction few days after surgery. If child shows no sign of improvement or inadequate rhythm recovery within 10-14 days postsurgery then permanent pacemaker should be considered.

\section{CONCLUSION}

In summary, arrhythmias are common after pediatric cardiac surgery. JET is the most common type of tachyarrhythmia seen during early postoperative care. Postoperative arrhythmias can be life-threatening tachyarrhythmia and need to be recognized and managed immediately. Majority of children recover with time and proper management. On the other hand, heart block is common bradyarrhythmia encountered postsurgery that may require temporary pacing during postoperative period. Most of children recover within 1-2 weeks postsurgery. Some patients may continue to exhibit abnormal slow rhythm and depends on temporary pacemaker after 10-14 days postsurgery. Permanent pacemaker would be recommended and indicated in these cases to support cardiac rhythm and prevent any hemodynamic embarrassment.

\section{Financial support and sponsorship}

Nil.

\section{Conflicts of interest}

There are no conflicts of interest.

\section{REFERENCES}

1. Talwar S, Patel K, Juneja R, Choudhary SK, Airan B. Early postoperative arrhythmias after pediatric cardiac surgery. Asian Cardiovasc Thorac Ann 2015;23:795-801.

2. Delaney JW, Moltedo JM, Dziura JD, Kopf GS, Snyder CS. Early postoperative arrhythmias after pediatric cardiac surgery. J Thorac Cardiovasc Surg 2006;131:1296-300.

3. Grosse-Wortmann L, Kreitz S, Grabitz RG, Vazquez-Jimenez JF, Messmer BJ, von Bernuth G, et al. Prevalence of and risk factors for perioperative arrhythmias in neonates and children after cardiopulmonary bypass: Continuous holter monitoring before and for three days after surgery. J Cardiothorac Surg 2010;5:85.

4. Anselmi A, Abbate A, Girola F, Nasso G, Biondi-Zoccai GG, Possati G, et al. Myocardial ischemia, stunning, inflammation, and apoptosis during cardiac surgery: A review of evidence. Eur J Cardiothorac Surg 2004;25:304-11.

5. Skippen PW, Sanatani S, Gow RM, Froese N. Diagnosis of postoperative arrhythmias following paediatric cardiac surgery. Anaesth Intensive Care 2009;37:705-19.

6. Polderman KH, Girbes AR. Severe electrolyte disorders following cardiac surgery: A prospective controlled observational study. Crit Care 2004;8:R459-66.

7. Valsangiacomo E, Schmid ER, Schüpbach RW, Schmidlin D, Molinari L, Waldvogel $\mathrm{K}$, et al. Early postoperative arrhythmias after cardiac operation in children. Ann Thorac Surg 2002;74:792-6.

8. He D, Sznycer-Taub N, Cheng Y, McCarter R, Jonas RA, Hanumanthaiah S, et al. Magnesium lowers the incidence of postoperative junctional ectopic tachycardia in congenital heart surgical patients: Is there a relationship to surgical procedure complexity? Pediatr Cardiol 2015;36:1179-85.

9. Verma YS, Chauhan S, Gharde P, Lakshmy R, Kiran U. Role of magnesium in the prevention of postoperative arrhythmias in neonates and infants undergoing arterial switch operation. Interact Cardiovasc Thorac Surg 2010;11:573-6.

10. Kleinman ME, Chameides L, Schexnayder SM, Samson RA, Hazinski MF, Atkins DL, et al. Part 14: Pediatric advanced life support: 2010 American Heart Association Guidelines for Cardiopulmonary Resuscitation and Emergency Cardiovascular Care. Circulation 
2010;122 18 Suppl 3:S876-908.

11. Batra AS, Balaji S. Post operative temporary epicardial pacing: When, how and why? Ann Pediatr Cardiol 2008;1:120-5.

12. Cools E, Missant C. Junctional ectopic tachycardia after congenital heart surgery. Acta Anaesthesiol Belg 2014;65:1-8.

13. Haas NA, Plumpton K, Justo R, Jalali H, Pohlner P. Postoperative junctional ectopic tachycardia (JET). Z Kardiol 2004;93:371-80.

14. Klautz RJ, Baan J, Teitel DF. Contribution of synchronized atrial systole to left ventricular contraction in the newborn pig heart. Pediatr Res 1998;43:331-7.

15. Abdelaziz O, Deraz S. Anticipation and management of junctional ectopic tachycardia in postoperative cardiac surgery: Single center experience with high incidence. Ann Pediatr Cardiol 2014;7:19-24.

16. Laird WP, Snyder CS, Kertesz NJ, Friedman RA, Miller D, Fenrich AL. Use of intravenous amiodarone for postoperative junctional ectopic tachycardia in children. Pediatr Cardiol 2003;24:133-7.

17. Kadam SV, Tailor KB, Kulkarni S, Mohanty SR, Joshi PV, Rao SG. Effect of dexmeditomidine on postoperative junctional ectopic tachycardia after complete surgical repair of tetralogy of Fallot: A prospective randomized controlled study. Ann Card Anaesth 2015;18:323-8.

18. Chrysostomou C, Sanchez-de-Toledo J, Wearden P, Jooste EH, Lichtenstein SE, Callahan PM, et al. Perioperative use of dexmedetomidine is associated with decreased incidence of ventricular and supraventricular tachyarrhythmias after congenital cardiac operations. Ann Thorac Surg 2011;92:964-72.

19. Adamson PC, Rhodes LA, Saul JP, Dick M 2 ${ }^{\text {nd }}$, Epstein MR, Moate P, et al. The pharmacokinetics of esmolol in pediatric subjects with supraventricular arrhythmias. Pediatr Cardiol 2006;27:420-7.

20. Saul JP, Scott WA, Brown S, Marantz P, Acevedo V, Etheridge SP, et al. Intravenous amiodarone for incessant tachyarrhythmias in children: A randomized, double-blind, antiarrhythmic drug trial. Circulation 2005;112:3470-7.

21. Kardelen F, Celiker A, Ozer S, Ozme S, Oto A. Sinus node dysfunction in children and adolescents: Treatment by implantation of a permanent pacemaker in 26 patients. Turk J Pediatr 2002;44:312-6.

22. Pace Napoleone C, Mariucci E, Angeli E, Oppido G, Gargiulo GD. Sinus node dysfunction after partial anomalous pulmonary venous connection repair. J Thorac Cardiovasc Surg 2014;147:1594-8.

23. Ayyildiz P, Kasar T, Ozturk E, Ozyilmaz I, Tanidir IC, Guzeltas A, et al. Pacing evaluation of permanent or transient complete heart block after open heart surgery for congenital heart disease. Pacing Clin Electrophysiol 2016;39:160-5.

24. Graham TP Jr., Bernard YD, Mellen BG, Celermajer D, Baumgartner H, Cetta F, et al. Long-term outcome in congenitally corrected transposition of the great arteries: A multi-institutional study. J Am Coll Cardiol 2000;36:255-61. 